\title{
Makenzy Orcel, Maître-Minuit
}

\section{Roberto Ferraroni}

\section{Q OpenEdition}

\section{Journals}

\section{Edizione digitale}

URL: https://journals.openedition.org/studifrancesi/21829

DOI: 10.4000/studifrancesi.21829

ISSN: 2421-5856

\section{Editore}

Rosenberg \& Sellier

\section{Edizione cartacea}

Data di pubblicazione: 1 décembre 2019

Paginazione: 620-621

ISSN: 0039-2944

\section{Notizia bibliografica digitale}

Roberto Ferraroni, «Makenzy Orcel, Maitre-Minuit», Studi Francesi [Online], 189 (LXIII | III) | 2019, online dal 01 mars 2020, consultato il 11 novembre 2021. URL: http://journals.openedition.org/studifrancesi/ 21829 ; DOI: https://doi.org/10.4000/studifrancesi.21829

Questo documento è stato generato automaticamente il 11 novembre 2021 .

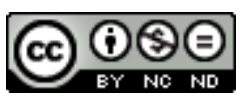

Studi Francesi è distribuita con Licenza Creative Commons Attribuzione - Non commerciale - Non opere derivate 4.0 Internazionale. 


\title{
Makenzy Orcel, Maître-Minuit
}

\author{
Roberto Ferraroni
}

\section{NOTIZIA}

Makenzy Orcel, Maître-Minuit, Paris, Zulma, 2018, 310 pp.

1 Ammanettato ad un letto d'ospedale, Poto, protagonista dell'ultimo romanzo dello scrittore haitiano Orcel, si racconta ad uno sconosciuto che si trova a condividere con lui la camera. Partendo dalla sua infanzia, proseguendo col difficile rapporto/non rapporto con la madre, che forse non era nemmeno la madre naturale, la loro separazione, l'incontro con MOI, che lo salverà da una vita di stenti diventando la sua guida, il protagonista si svela al lettore arrivando a raccontare della sua grande arte di disegnatore, che diventerà una vera e propria professione, grazie alla quale potrà sopravvivere.

Quello che ad una lettura superficiale potrebbe sembrare un romanzo biografico, si rivela in realtà qualcosa di diverso, che nasconde molto di più. Nell'arco dei cinquant'anni che vengono descritti, infatti, il lettore non impara soltanto a conoscere la storia dell'io narrante, ma viene messo in diretto contatto con la realtà haitiana e i cambiamenti che la società ha subito in quell'arco temporale. L'immaginario dittatore Papa-à-vie, per esempio, non è altro che una (non troppo) velata trasposizione letteraria di François Duvalier, che per anni ha vessato l'isola col suo governo. Le vicissitudini di Poto sono completamente intrecciate con la realtà di quegli anni, a tal punto che il lettore ha la possibilità di sentirsi pienamente immerso in un mondo con cui, molto probabilmente, non ha avuto la possibilità di entrare direttamente in contatto. Orcel non fa tuttavia trasparire solo la cupa e opprimente atmosfera degli anni bui della dittatura, ma anche il clima carico di tensione e paura del periodo che è seguito alla caduta di Duvalier, in cui le condizioni di vita degli Haitiani non hanno goduto di sufficienti miglioramenti. Il lettore si trova a condividere le emozioni del protagonista e al contempo a provare un senso di oppressione e soffocamento nel vivere, leggendo, come un abitante dell'isola di quegli anni. 
Il romanzo è diviso in quattro parti, precedute da poche pagine iniziali prive di titolo $\mathrm{e}$ seguite da una brevissima conclusione intitolata "Enfin". Ogni capitolo serve a definire una fase ben precisa della vita di Poto, a partire dal primo che prende il titolo "Marie Élitha Démosthène Laguerre", ossia il nome della madre (presunta) di Poto. È interessante che venga intitolato un intero capitolo alla donna, quasi a volerle dare la parola. Interessante e strano allo stesso tempo, in quanto per tutta la durata del romanzo in cui è presente, non le viene praticamente mai data la possibilità di parlare, di giustificare le proprie azioni. Il ritratto di Marie Élitha, nominata sempre rigorosamente con il nome per esteso, quasi ad affermare un'identità che altrimenti parrebbe eterea al lettore, è indubbiamente negativo, di una donna tossicodipendente, distaccata dalla realtà, che non si cura del suo stesso figlio.

4 I capitoli seguenti toccano momenti diversi della vita di Poto, che cresce e diventa uomo, vive prima di stenti, fingendosi pazzo, e impara poi a valorizzarsi, anche grazie all'aiuto di MOI, suo amico per molti anni. Il nome della madre ritornerà ancora varie volte, come a sottolineare che quel rapporto, nonostante si sia interrotto, continua ad avere un peso importante nella vita del protagonista.

5 Il romanzo coinvolge e "opprime" non solo dal punto di vista contenutistico, ma anche grazie alla bravura di Orcel che con un ritmo incalzante fornisce descrizioni dettagliate del mondo haitiano di quegli anni. L'atmosfera cupa e pesante è trasmessa fin da subito anche da un punto di vista visivo e grafico: pochissimi "a capo", dialoghi inseriti nella narrazione senza "virgolette" o altri segni di punteggiatura che aiutino il lettore ad individuarli, nessuna lettera maiuscola, nemmeno nei titoli, con la sola eccezione delle iniziali dei nomi propri e di MOI, scritto sempre interamente in maiuscolo, probabilmente per sottolineare il suo valore e il suo distinguersi dal resto degli Haitiani, nonché l'importanza del personaggio per il protagonista. Se da un lato quindi lo stile crea un'atmosfera ancora più greve di quanto le parole e il contenuto non riescano a fare da soli, dall'altro regala al romanzo una forma poetica difficilmente riscontrabile in altri autori haitiani contemporanei. La poesia del testo sta nella scelta dei termini, nel modo in cui vengono disposti nelle frasi, ma talvolta addirittura nella disposizione grafica dei periodi: in alcuni punti della narrazione, Orcel abbandona lo stile incalzante per dividere le frasi in versi secondo le regole metriche della poesia tradizionale (p. 36), o addirittura sovvertendo le regole della poesia classica, quasi un moderno futurista (p. 30).

6 Ma chi è il Maître-Minuit che dà il titolo al libro? Non è un personaggio che incontriamo nella storia, ma un'antica figura della mitologia haitiana, conosciuta da ogni abitante dell'isola: «c'est un homme qui reste debout, avance toujours, quoi qu'il arrive» (p. 24), risponde Grann Julienne al piccolo Poto, quando questi la interroga su questa entità che nessuno ha mai visto. Si dice che abbia le dimensioni di un gigante $\mathrm{e}$ che con un solo passo possa scavalcare una città intera; continua a camminare, non si ferma mai. Si può quasi pensare che Orcel voglia sussurrare al lettore un parallelismo nascosto tra Maître-Minuit "qui reste debout" e Poto, che non si fa mai abbattere, cade e si rialza sempre, non viene mai completamente piegato da ciò che di negativo gli accade.

7 La maestria dell'autore in questo romanzo emerge con vigore da ogni pagina, da ogni parola. Orcel affascina con la sua forza espressiva, con il suo stile ritmato, con la sua prosa che a tratti tocca e si intreccia con la poesia. Il lettore arriva all'ultima pagina desideroso di conoscere il destino di Poto, portando con sé un'accresciuta conoscenza 
della cultura haitiana e soprattutto una consapevolezza della realtà dell'isola, vissuta, anno dopo anno, attraverso le parole del protagonista. 